\title{
Combined abdominoplasty with scar-less open cholecystectomy
}

\begin{abstract}
When abdominoplasty is desired, special considerations must be taken in Patients with previous abdominal scars. Open cholecystectomy scar is a relative contraindication for abdominoplasty, due to extensive abdominal flap undermining, which reaches higher up to the costal margins. In our patient the procedure of combined abdominoplasty and open cholecystectomy was completed without leaving any scar of open cholecystectomy, and this led to a better cosmetic results and viability of the whole abdominal wall skin with no complications from both procedures.
\end{abstract}

\section{KEYWORDS: abdominoplasty, scar-less, open cholecystectomy, combined procedures}

\section{Introduction}

Patients seeking Abdominoplasty show a list of common desires: a desire to decrease loose, draping skin, have a tighter, flatter abdomen and improve the abdominal contour [1].

In addition; operations on the abdominal wall like hernias may further weaken and partially denervate the musculo-aponeurotic system [2]. Typically if there is pronounced skin laxity; more than $6-8 \mathrm{~cm}$ of skin needs to be excised in the vertical dimension. Extensive undermining is necessary to provide exposure to a lax abdominal wall and to allow redraping of the skin [2]. Supra-umbilical incisions such as subcostal incisions for cholecystectomy pose a greater risk for superior skin flap survival. In these cases a limited skin undermining should be chosen to preserve adequate perforating vessels to the skin flap below the transverse incisions [3].

Patients with previous abdominal scars may require special considerations if abdominoplasty is contemplated. A subcostal, open cholecystectomy scar can contraindicate a traditional abdominoplasty, with its extensive abdominal flap undermining, which reaches up to the costal margins and xiphoid [4]. The only feeding vessels to the elevated abdominal flap in that instance consist of the lateral intercostals, subcostal, and lumbar perforator vessels, which course anteriorly in the fat superficial to Scarpa's fascia. These vessels are interrupted by a subcostal scar and this can lead to abdominal flap necrosis inferomedial to the scar [5].

\section{Case Presentation}

Our patient is 45 years old heavy smoker male patient presented to our hospital with abdominal wall laxity after massive weight loss of about 30 kilograms.

He came seeking for a cosmetic procedure (abdominoplasty) to reduce the abdominal wall laxity and skin excess. During preoperative preparatory period, recurrent attacks of abdominal pain in right upper quadrant referred to his back and his right shoulder occurred and the patient also mentioned that he had previous attacks several times during the last 4 months.

Abdominal ultrasound examination was done and revealed chronic calcular cholecystitis with multiple gall bladder stones with nondilated common bile duct and no manifestations of jaundice. The initial plan of management of the patient was to combine both the abdominoplasty procedure with laparoscopic cholecystectomy. Unfortunately the medical center had no facility for laparoscopy operations and referral to other qualified centers was difficult and quite distant for the patient. Decision was taken to combine both procedures through one surgical incision and surgical consent was taken from the patient after discussing the risks of the combined procedures, after the patient insisted to postpone the cholecystectomy operation till complete healing of the abdominoplasty operations and use medications for cholecystitis till the estimated date of cholecystectomy operation.

Patient was in supine position, under general

\section{Mohamed Elsayed}

Mohamed $^{1 *}$ and

Sherif Hussein ${ }^{2}$

${ }^{1}$ Ahmed Maher Teaching Hospital

Cairo, Egypt

${ }^{2}$ Kasr Alini Medical school

*Author for correspondence:

mghabn5777@yahoo.com 
anesthesia, Lower Abdominal sea-gull incision was done .dissection and raising the skin and subcutaneous layer was done and the whole flap was elevated to the xiphoid process and the subcostal margins bilaterally. Reflection of the whole abdominal flap was done to expose the right subcostal area to reach the right subcostal edge and that was done by the plastic surgeon member of the team (FIGURE 1A and 1B). Right subcostal incision was performed through the right sided abdominal muscles down to the peritoneum. The gall bladder was delivered

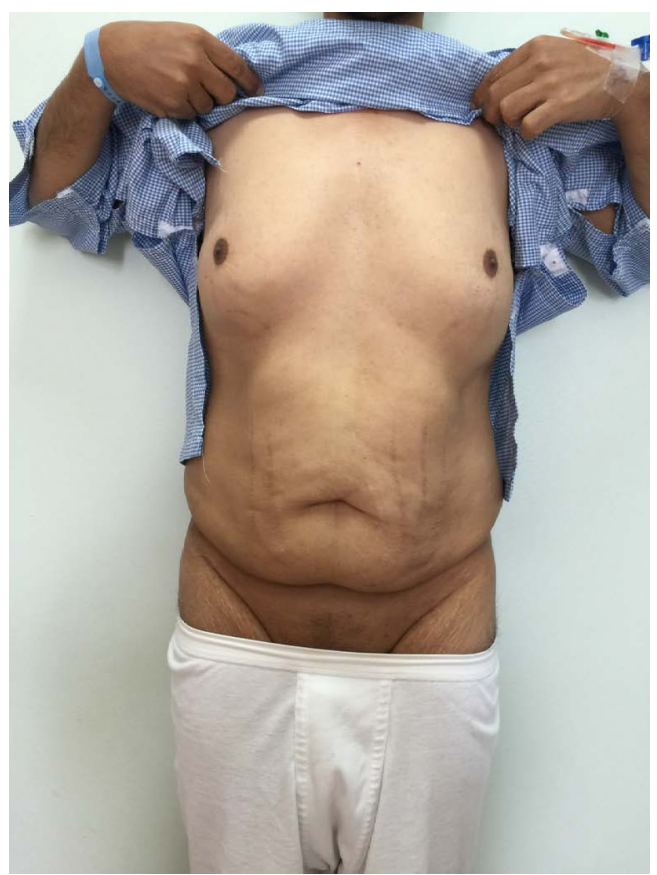

Figure 1 A. Pre-operative anterior view.

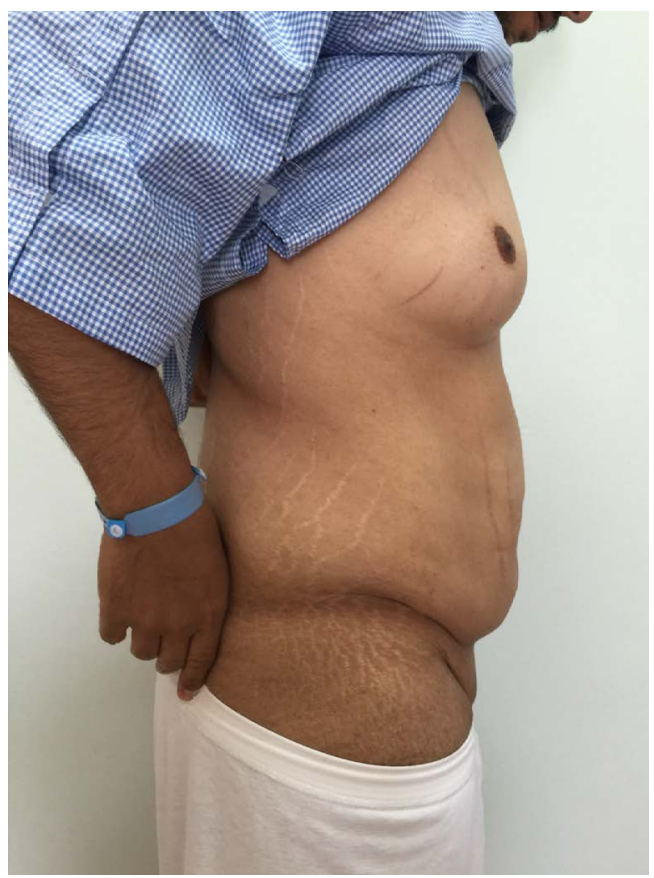

Figure 1B. Pre-operative lateral view. through the incision to be dissected from the bed and ligated then removed and hemostasis of the bed was assured through the incision with intra-peritoneal vacuum drain inserted through the right flank. Anatomical repair of the muscle layers was done by the general surgeon (FIGURE 2A-2C).

Dermolipectomy was continued as usual and excision of the excess skin and subcutaneous tissues with two subcutaneous drains inserted (FIGURE 2D) Blood loss was about $700 \mathrm{cc}$

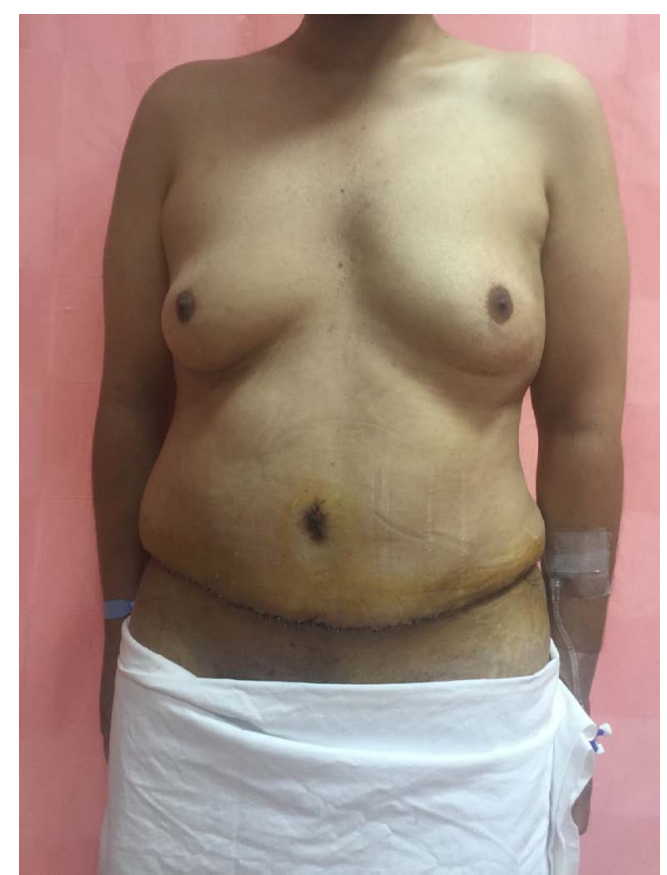

Figure 1C. Post-operative anterior view.

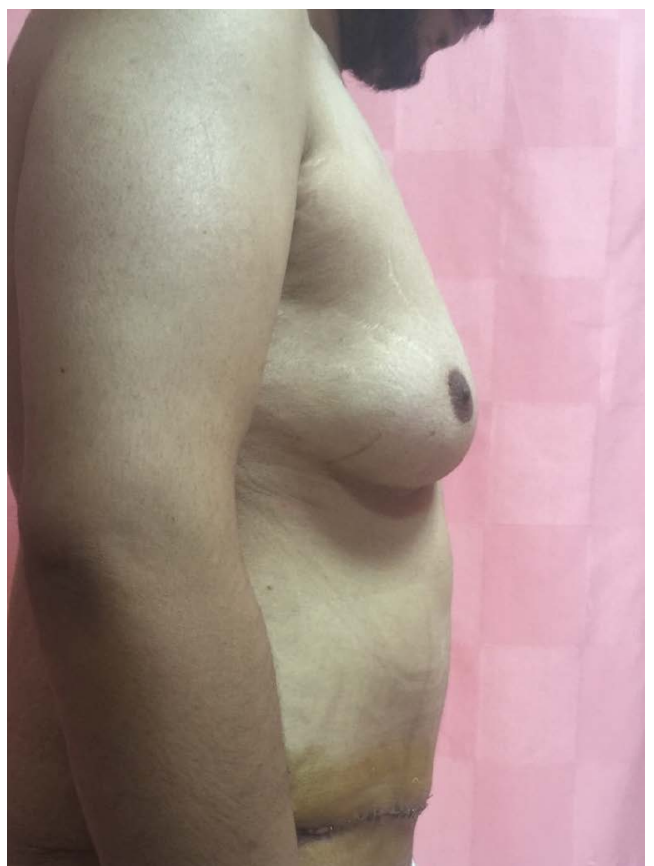

Figure 1D. Post-operative lateral view. 


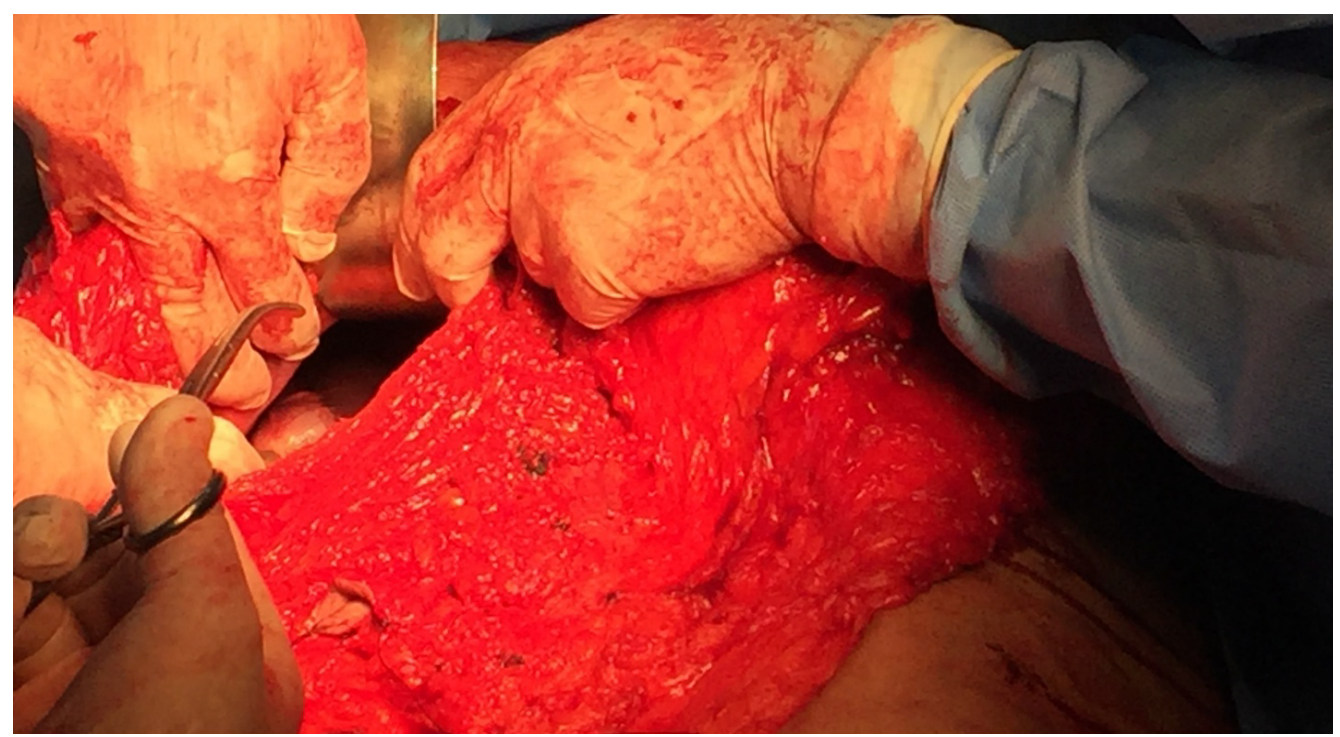

Figure 2A. Intra-operative with exposure of the gall bladder where the umbilical stump is visualized and the gall bladder is seen intra-abdominal.

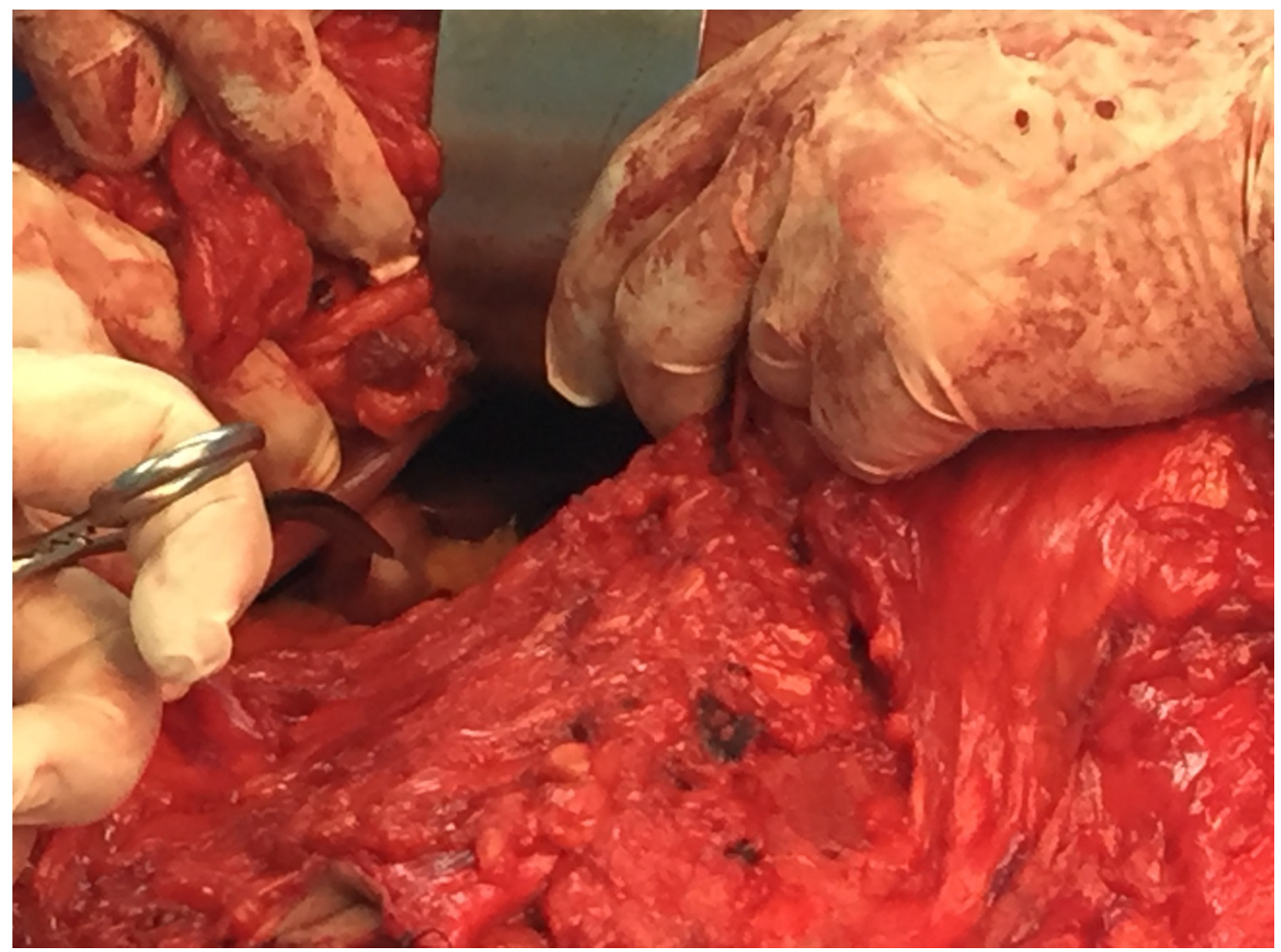

Figure 2B. Intra-operative with captured gall bladder intra abdominally.

with no intraoperative blood transfusion. The procedure of combined abdominoplasty and open cholecystectomy was completed without leaving any signs of open cholecystectomy, and this led to a better cosmetic result from the patients' point of view (FIGURE 1C and 1D). There were no complication from both procedures, and full ambulation was obtained post operatively.

\section{Discussion}

The combined abdominoplasty with cholecystectomy was done in 3 cases laparoscopically in 2012 with no complications and this led to a better cosmetic result from the patients' point of view [6].

In another study of one hundred three patients underwent abdominoplasty combined 


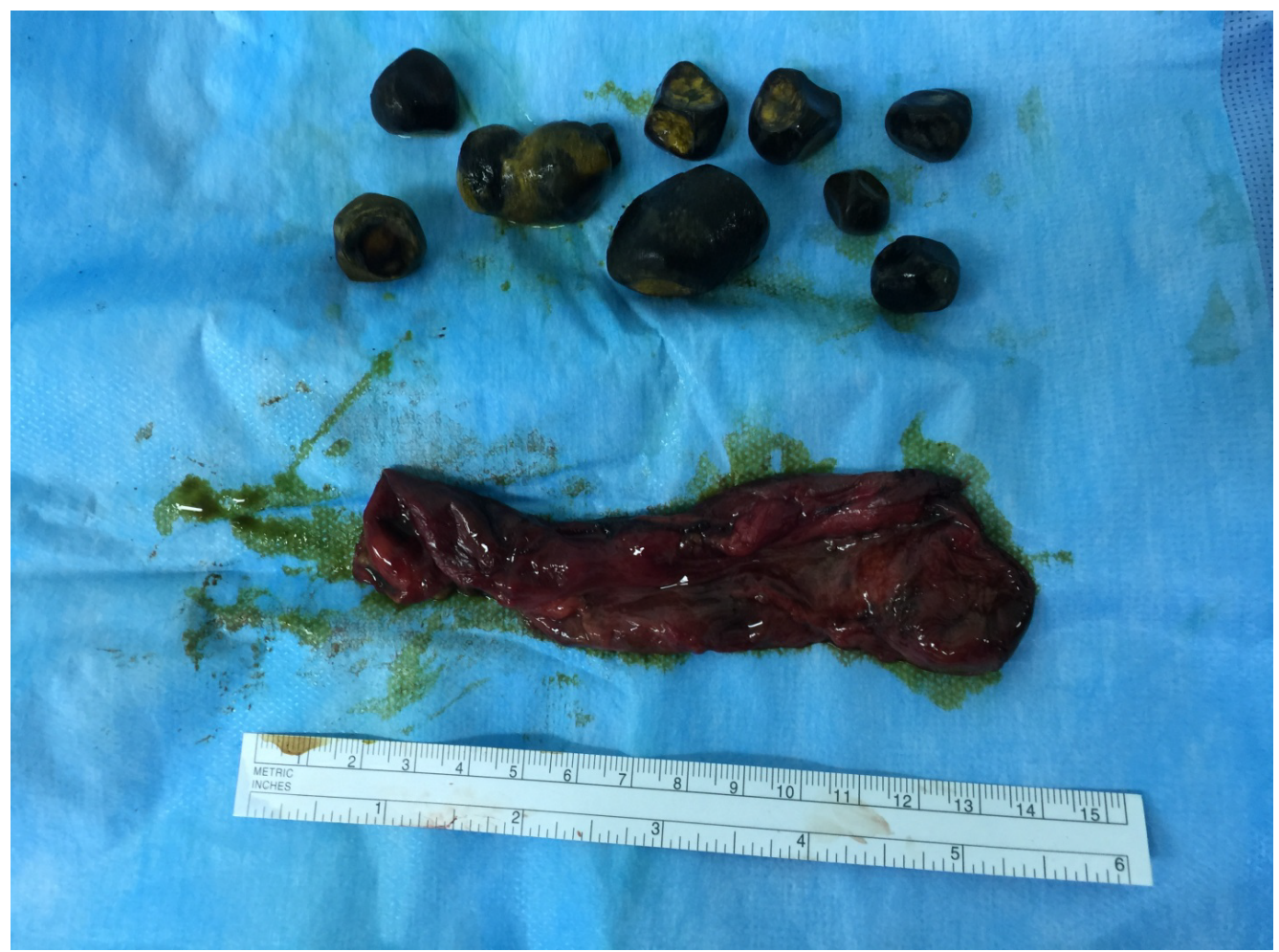

Figure 2C.Gall bladder opened and extracted gall bladder stones

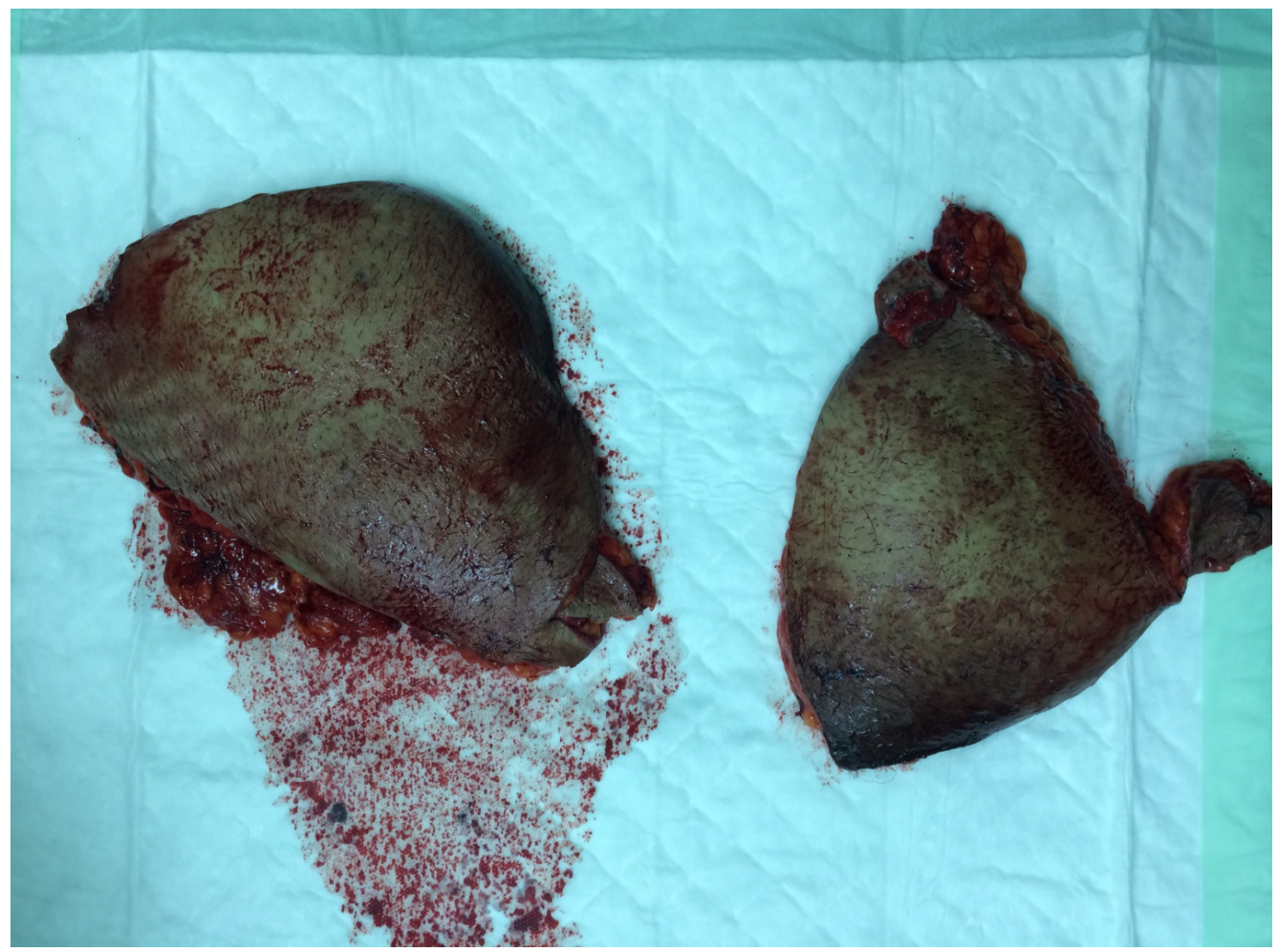

Figure 2D. The excised pannus (two parts).

with other intra-abdominal procedures including 67 tubal ligations, 34 total abdominal hysterectomies and 2 cholecystectomies, from January 1983 to July 1991 and concluded that good surgical teams can safely and effectively combine abdominoplasty with intra-abdominal procedures [7].

In our case, there was no facility to do combine laparoscopic cholecystectomy and abdominoplasty; it was also so difficult to refer 
the patient to other center. The patient agreed to do the combined procedure in an open cholecystectomy way without laparoscopy. The procedure was done with no complications and passed smoothly post operatively. The aesthetic and functional outcomes were greatly satisfactory for both the patient and the doctors.

\section{Informed Consent}

Written consent for release of the patient's historical and photographic information was obtained before the time of the operation. 


\section{REFERENCES}

Psillakis JM. Plastic surgery of the abdomen with improvement in the body contour. Clin Plast Surg 11,465477 (1984).

Pitanguy I. Dermatolipectomy of the abdominal wall, thighs, buttocks and upper extremity. Converse JM (ed.) Reconstructive plastic surgery. WB Saunders, Philadelphia, PA, pp 38003823 (1977).

Bozola AR, Psillakis JM. Abdominoplasty: A new concept and classification for treatment. Plast Reconstr 82,983-993 (1988).
Lockwood T. High-lateral tension abdominoplasty with superficial fascial system suspension. Plast Reconstr Surg 96,603 (1995).

Lockwood T. Maximizing aesthetics in lateral-tension abdominoplasty and body lifts. Clin Plast Surg 31,523 (2004).

Prasad MB, Surapaneni SR, Dabade SS. Scarless cholecystectomy: laparoscopic cholecystectomy with abdominoplasty. Indian J Surg 74(6), 4868 (2012).

Gemperli R, Neves RI, Bonamichi GT, et al. Abdominoplasty combined with other intra-abdominal procedures. Ann Plast Surg 29(1),1822 (1992). 